\title{
High Resolution Shear Profile Measurements in Entangled Polymers
}

\author{
Keesha A. Hayes, ${ }^{1}$ Mark R. Buckley, ${ }^{2}$ Itai Cohen, ${ }^{2}$ and Lynden A. Archer ${ }^{1}$ \\ ${ }^{1}$ School of Chemical \& Biomolecular Engineering, Cornell University, Ithaca, New York 14853, USA \\ ${ }^{2}$ Department of Physics, Cornell University, Ithaca, New York 14853, USA
}

(Received 21 May 2008; published 17 November 2008)

\begin{abstract}
We use confocal microscopy and particle image velocimetry to visualize motion of 250-300 $\mathrm{nm}$. fluorescent tracer particles in entangled polymers subject to a rectilinear shear flow. Our results show linear velocity profiles in polymer solutions spanning a wide range of molecular weights and number of entanglements ( $8 \leq Z \leq 56$ ), but reveal large differences between the imposed and measured shear rates. These findings disagree with recent reports that shear banding is a characteristic flow response of entangled polymers, and instead point to interfacial slip as an important source of strain loss.
\end{abstract}

Flow properties of entangled polymers are important in myriad commercial processes for molding, extruding, and spinning plastic components. The Doi-Edwards (DE) theory provides the most successful molecular framework for understanding these properties. Developed around the reptation or "tube" model [1], this theory contends that a melt of entangled polymers responds affinely to instantaneous macroscopic deformations. The affine response is sustained by long-lived entanglements between molecules and causes the network of entanglements (tube) constraining any given molecule to orient and stretch in the same way as does the macroscopic melt. Polymer molecules trapped in the tube initially stretch and orient in synergy with their environment.

DE predictions for step strain, oscillatory, and steady shear flows agree, sometimes quantitatively, with experiments [2-6]. A more controversial prediction is that under steady shear, the shear stress is a multivalued function of the imposed shear rate. Thus, simple shear flow is unstable to perturbations in shear rate and should produce shear banding $[7,8]$. Surprisingly, with the exception of entangled wormlike micellar fluids [9-11], banding is generally not observed in flows of entangled polymers at any shear rate. This implies that some other dynamic processes not taken into account by the theory must contribute to the fluid's response. Efforts to date have focused on understanding how convective acceleration of reptation [12-17], tube diameter shrinkage [18], and slip [19-22] near the shearing surfaces influence this prediction. All three processes eliminate or weaken the driving force for entangled polymers to shear band and, when integrated into the DE theory, lead to steady shear stress predictions that compare favorably with experiments using moderately entangled polymers.

Recent velocity profile measurements using $10 \mu \mathrm{m}$ particles dispersed in entangled polybutadiene solutions show, for the first time, that entangled polymer systems do in fact appear to shear band [23-25]. Surprisingly, these studies find that shear banding occurs even in solutions with intermediate levels of entanglements, generally thought to be well described by DE theory with the aforementioned modifications. Parameters such as shear strain and shear rate, widely used to characterize shear of entangled polymers, are ill-defined in a banded fluid, implying that all published nonlinear rheological data on these systems are challenged by the recent findings. Establishing whether these recent observations of banding are generally applicable to polymers in the class entangled fluids is therefore of critical significance to the field [26].

In this Letter we report on a technique that incorporates fast confocal microscopy to investigate flow of entangled polybutadiene solutions. This technique has previously been shown to accurately detect shear banding in colloidal suspensions on the micron scale [27]. We apply it to analyze rectilinear shear flow between parallel planes because this geometry has multiple inherent advantages over the more commonly used Couette and cone-and-plate shear geometries. First, the absence of curvature in material lines makes interpretation of the velocity profile straightforward. Second, polymeric materials are not susceptible to any of the plethora of hydrodynamic, elastic, and edge effects that plague fast flow experiments of these fluids [28-31]. Additionally, for low enough gap (plane separation, $H$ ) to width $(W)$ ratios $H / W=a_{s}<1 / 50$, weaker secondary flows induced by normal stresses are suppressed [32]. Finally, the temperature rise $(\Delta T)$ produced during shear of a low thermal-conductivity $(k)$ polymer fluid can have profound effects on the velocity profile in any flow geometry [33]. For our flow geometry, $\Delta T=\frac{\sigma \dot{\gamma}}{k} H^{2}$, where $\sigma$ and $\dot{\gamma}$, are, respectively, the shear stress and shear rate. Thus, provided the gap is small, flow anomalies produced by viscous heating can be minimized. The experiments we report are performed in a custom-built shear cell with $a_{s} \leq$ $1 / 143$ and $H=25-35 \mu \mathrm{m}$; both are at least 1 order of magnitude lower than is typical for polymer flow experiments. Our device therefore provides an essentially ideal setting for investigating banding in polymer flows. Remarkably, we find that the measured velocity profiles 
are generally linear at shear rates well into the nonNewtonian flow regime.

According to the DE theory, the shear rates at which shear stress, $\sigma_{y x} \equiv \sigma_{s}$ (flux of momentum normal to the flow direction, $x$ ), is multivalued are related to the relaxation mechanisms accessible to polymers within their local tubes. At low rates, $\dot{\gamma}<\tau_{\text {rept }}^{-1}$, polymer chains escape their surroundings by reptation essentially unperturbed by the shear. As a result, momentum is transferred by secondary bonds and entanglements between randomly oriented molecular units, leading to a Newtonian response. For shear rates $\dot{\gamma} \geq \tau_{\text {rept }}^{-1}$, chains slip within their affinely deforming tubes to preserve their length, but cannot otherwise escape the tube constraint. Consequently, the trapped chains orient in the direction of shear, providing progressively less efficient momentum transport between fluid layers as shear rate increases. The resultant steady-state $\sigma_{s}$-shear-rate relationship can be expressed analytically,

$$
\begin{aligned}
\sigma_{s}\left(\dot{\gamma} \ll \tau_{\text {Rouse }}^{-1}\right)= & \int_{-\infty}^{t} \sigma_{s}\left(\left\{t-t^{\prime}\right\}, \dot{\gamma}\left\{t-t^{\prime}\right\}\right) d t^{\prime} \\
& \approx \frac{15}{4} \dot{\gamma} \tau_{\text {rept }} G_{e} /\left[1+\frac{4}{15}\left(\dot{\gamma} \tau_{\text {rept }}\right)^{2}\right],
\end{aligned}
$$

and manifests a maximum at $\dot{\gamma}=\sqrt{15 / 4} \tau_{\text {rept }}^{-1}$. Here $G_{e}$ is the plateau modulus of the polymer. The DE theory predicts that shear alignment continues until $\dot{\gamma} \tau_{\text {Rouse }} \geq 1$, at which point polymer chains begin to deform/stretch in sympathy with their surroundings. Chain stretch makes a contribution $\dot{\gamma} \tau_{\text {Rouse }} G_{N}$ to $\sigma_{s}$, which arrests the downturn in $\sigma_{s}$ (Fig. 1). In the multivalued regime, $\sqrt{15 / 4} \tau_{\text {rept }}^{-1} \leq$ $\dot{\gamma}<\tau_{\text {Rouse }}^{-1}$, the material forms two bands, each characterized by different shear rates and distinct relaxation processes.

To characterize the velocity profile, $v(y), 250-300 \mathrm{~nm}$ core-shell silica tracer particles, which encapsulate the fluorophore tetramethyl rhodamine isothiocyanate were

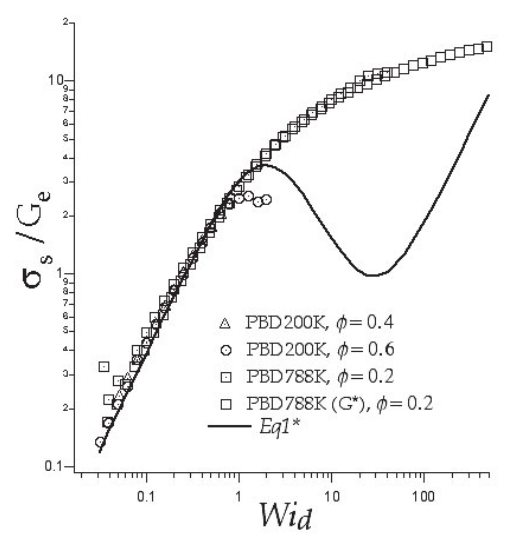

FIG. 1. Dimensionless steady-state shear stress versus dimensionless shear rate for PBD solutions. The open squares represent the dimensionless complex modulus $G^{*}$ versus frequency for PBD788K, $\phi=0.2$, from LVE measurements. dispersed in polybutadiene (PBD) solutions. The average number of entanglements $Z$ was varied by changing the volume fraction of two high-molar-mass polymers $\bar{M}_{w}=$ $7.9 \times 10^{5} \mathrm{~g} / \mathrm{mol}, \quad \bar{M}_{w} / \bar{M}_{n}=1.1 \quad(\mathrm{PBD} 788 \mathrm{~K}$, Polymer Source, Inc.) and $\bar{M}_{w}=2 \times 10^{5} \mathrm{~g} / \mathrm{mol}, \bar{M}_{w} / \bar{M}_{n}=1.05$, (PBD200K, synthesized in-house) in an unentangled PBD host, $\bar{M}_{w}=1 \times 10^{3} \mathrm{~g} / \mathrm{mol}$. The seeded solutions were sandwiched between glass planes maintained at a separation $H$ between 25 and $35 \mu \mathrm{m}$. The plates were integrated into a motorized planar-Couette shear cell, which fits on the sample stage of a Zeiss 5-Live inverted confocal microscope equipped with a $100 \times$ objective and $120 \mathrm{fps}$ maximum capture rate. Because the tracer particles are substantially smaller than ones used previously [23-26], the velocity profile can be probed on a finer scale.

To generate shear flow in the polymer, one plane is moved relative to the other at a prescribed velocity $V \boldsymbol{e}_{x}$ using a controlled-speed inchworm motor (Burleigh-TSE 820). The nominal shear rate, $\dot{\gamma}_{\text {nom }}=V / H$, is varied by adjusting $V$ and the shear force, $F_{s}$, measured using a singlepoint load cell (S300, Strain Measurement Devices, Inc.). The entire velocity profile is characterized, one layer at a time (typically $3 \mu \mathrm{m}$ apart), using the confocal microscope to scan two-dimensional micrographs. To construct the profile, we simultaneously monitor $\sigma_{s}(t)=F_{s}(t) / W^{2}$ versus time and record the time-dependent particle motions in a given layer. A modified version of MATPIV is used to extract the displacement values. At steady state, defined here as the time at which $\sigma_{s}$ and the tracer velocity cease to vary with time, the average particle velocity in that layer is determined. This process is repeated layer-by-layer until the profile is characterized.

Figure 1 reports baseline steady-state shear stress-versus shear rate data for all polymers used in the study, obtained using an ARES controlled-strain rotational rheometer with cone-and-plate fixtures. The solid line in the figure is the prediction of Eq. (1) for a polymer with $Z=20$. For the PBD200K solutions, $\sigma_{s}$ and $\dot{\gamma}$ are nondimensionalized using the respective plateau moduli $G_{N} \approx G_{e}$ and disengagement times $\tau_{d} \approx \tau_{\text {rept }}$ deduced from linear viscoelasticity (LVE) measurements. For the PBD788K solution, the shear rate is nondimensionalized using $\tau_{d}$, but the shear stress requires a value $G_{e}=1.8 \times 10^{3} \mathrm{~Pa}$, which is about 25 times lower than $G_{N}$, to yield a stress-versus-rate curve consistent with the other fluids. This last observation is unusual for an entangled polymer solution, but has been confirmed using the frequency-dependent complex modulus $G^{*}$ data from LVE (open squares). Additionally, these $G_{N}$ values have been compared with $G_{N 0}$ for the PBD788K melt to verify the solution concentration, $\phi=$ $\left(G_{N} / G_{N 0}\right)^{3 / 7}$.

Figure 1 shows that most features predicted by the DE theory for $\dot{\gamma}<\tau_{d}^{-1}$, including the universal relationship between $\sigma_{s} / G_{e}$ and the dimensionless shear rate, $W i_{d} \equiv$ $\dot{\gamma} \tau_{d}$, are consistent with the experimental data. At higher 
shear rates, both PBD200K solutions display stress plateau regimes. Data in this regime are limited, however, because $\sigma_{s}$ displays erratic time-dependent behavior and consistent steady-state values could not be obtained. This behavior is observed in a $W i_{d}$ range where the DE model predicts multivalued stresses, implying either that the flow is banded or that other phenomena, e.g., slip or viscous heating, compromise the measurements. This should be contrasted with $\sigma_{s}$ versus $W i_{d}$ for the PBD788K solution, which shows no evidence of a plateau.

Figures 2(a)-2(e) provide a sample set of velocity profiles at shear rates spanning the simple shear, $\dot{\gamma}<\tau_{d}^{-1}$, banded shear flow, and high-shear stable shear flow, $\dot{\gamma}>$ $\tau_{\text {Rouse }}^{-1}$, predictions of the DE theory. $Z$ ranges from 8 to 56 , and the shear viscosity differs by more than three orders. The true shear rate, $\dot{\gamma}_{T}$, corresponding to the line slope $m$, and $\dot{\gamma}_{\text {nom }}=(V / H)_{\text {nom }}$ are provided in each case. It is apparent that the velocity profiles are in most cases fitted well by straight lines, showing no evidence of banding. Indeed at the highest rates in Figs. 2(c) and 2(d), $\dot{\gamma} \tau_{d}=5.8$
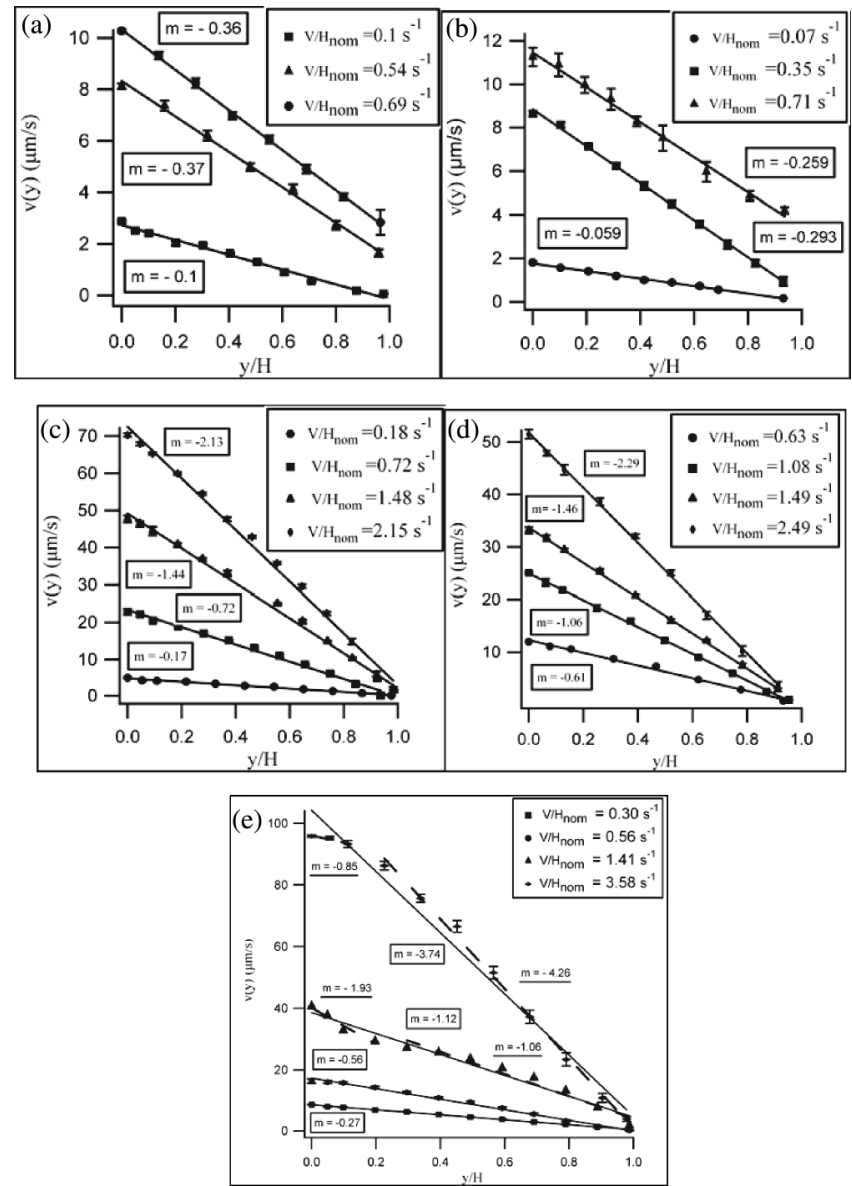

FIG. 2. Velocity profiles for PBD solutions, $y / H=0$ and 1 are the bottom and top plates, respectively. (a) PBD200K, $\phi=0.4$, $Z=32$; (b) PBD200K, $\phi=0.6, Z=56$; (c) PBD788K, $\phi=$ $0.05, Z=8$; (d) PBD788K, $\phi=0.1, Z=20$; (e) PBD788K, $\phi=0.2, Z=41$ and 27 , the profiles are decidedly linear. These shear rates are well into the non-Newtonian regime (Fig. 1) and substantially above those where shear banding is expected. Surprisingly, a solution with comparable levels of entanglement to PBD788K, $\phi=0.1$ was shown by Tapadia et al. [23] to shear band, which is inconsistent with our measurements.

The nominal and true shear rates are generally in excellent accord at low rates, $\dot{\gamma}_{\text {nom }} \ll \tau_{\text {rept }}^{-1}$. Except for the most viscous solutions, $\dot{\gamma}_{T} \approx \dot{\gamma}_{\text {nom }}$ over the full range studied; for the PBD200K, $\dot{\gamma}_{T}$ becomes systematically

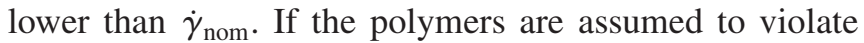
the no-slip condition equally at the two planes, the difference can be related to the slippage velocity, $V_{s}: \dot{\gamma}_{\text {nom. }}-$ $\dot{\gamma}_{T}=2 \frac{V_{s}}{H}$. Figure 3 plots $V_{s}$ against $\sigma_{s}$ for two PBD 200K solutions - both manifest two well-defined slip regimes. At stresses below a critical value $\sigma_{s}^{*} / G_{N} \approx 1.97$ and 1.3 , respectively, $V_{s}$ is an approximately linear function of $\sigma_{s}$. At higher $\sigma_{s}$, slip violations are much larger and $V_{s}$ increases rapidly. These observations are in good to excellent agreement with previous reports of interfacial slip in entangled polymers [21] based on other measurement techniques. Scaling theories [21-23] predict that slip by shear-induced disentanglement of surface tethered and bulk polymer chains exhibit multiple power-law regimes in $V_{s}$ versus $\sigma_{s}$, including the linear "Navier" slip regime observed here. These theories also predict a transition to large (macroscopic) slip velocities at $\sigma_{s}^{*} / G_{N} \approx 1$, which is also consistent with our experiments. Thus, we conclude that the stress plateau and erratic behavior seen in PBD200K solutions are not produced by bands, but by slip.

Figure 2(e) provides velocity profiles for a more entangled version of the PBD788K, $Z=51$ polymer.

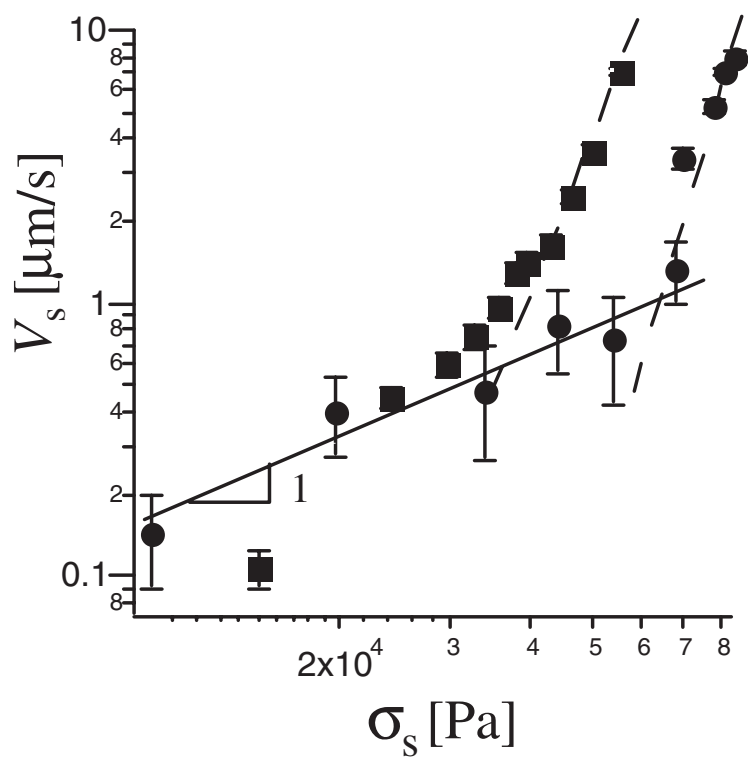

FIG. 3. Slip velocity versus shear stress for PBD200K solutions, $Z=32$ (squares), $Z=56$ (circles). 
Rheology measurements indicate that $\sigma_{s}$ versus $\dot{\gamma}$ is monotonic in the range where banding is predicted. Figure 2(e) shows that up to $\dot{\gamma} \tau_{d}=18 ; \dot{\gamma} \tau_{\text {rouse }} \approx 0.12$, the profile is linear, with slope $m$ close to the imposed shear rate, $(V / H)_{\text {nom. }}$. At $\dot{\gamma} \tau_{d}=45 ; \dot{\gamma} \tau_{\text {rouse }}=0.3, m$ begins to deviate from $(V / H)_{\text {nom }}$ and the velocity profile is weakly nonlinear in the near-wall region. A single straight line with $m<(V / H)_{\text {nom }}$ still fits the data, but the fit is poorer near the moving boundary; a combination of two lines yields a shear rate near the moving boundary roughly twice that in the bulk fluid. A similar result is observed for the highest rate studied $\left(\dot{\gamma} \tau_{d}=113 ; \dot{\gamma} \tau_{\text {rouse }}=0.74\right)$, with the apparent shear rate in the bulk now about $19 \%$ larger than $(V / H)_{\text {nom }}$. It could be argued that the steady-state velocity profiles for PBD788K, $Z=51$ are consistent with banding; however, the unusually large rates, $\dot{\gamma} \tau_{d} \approx Z$, at which the deviations from linearity are first seen rule out any straightforward explanation by DE theory. Indeed at such high shear rates, chain stretching and normal stresses are certainly appreciable making it impossible to ignore secondary flows, even in our carefully constructed device. Considering the range of $Z$, solution viscosities, and shear rates for which decidedly linear velocity profiles are observed, we conclude that shear banding is not a general feature of steady shear flows of entangled polymer liquids.

In conclusion, velocity profiles for entangled polymers in planar-Couette shear flow have been measured on the micron scale by combining confocal microscopy and velocimetry. Our results show that these profiles are generally linear for polymers with degrees of entanglement in the range 8 to 56 , and inconsistent with the notion that shear banding is a characteristic of entangled polymer flows. Our results also show that entangled polymer solutions violate the no-slip condition and that at shear stresses comparable to the elastic modulus, highly nonlinear slip behavior (strong slip) occurs.

This study was supported by the National Science Foundation (DMR0551185 and DMR0606040) and by KAUST-CU Center for Energy and Sustainability. We are grateful to Erik Herz and Ulrich Wiesner for providing fluorescent nanoparticle tracers used in the study.

[1] P. G. De Gennes, J. Chem. Phys. 55, 572 (1971).

[2] M. Doi and S.F. Edwards, The Theory of Polymer Dynamics (Oxford, New York, 1986).

[3] K. Osaki, Rheol. Acta 32, 429 (1993).

[4] E. V. Menezes and W. W. Graessley, J. Polym. Sci., Polym. Phys. Ed. 20, 1817 (1982).
[5] S. Baek, J. J. Magda, and R. G. Larson, J. Rheol. (N.Y.) 37, 1201 (1993).

[6] D. S. Pearson, Rubber Chem. Technol. 60, 439 (1987).

[7] J. Yerushalmi, S. Katz, and R. Shinnar, Chem. Eng. Sci. 25, 1891 (1970)

[8] G. Marrucci and N. Grizzuti, Gazz. Chim. Ital. 118, 179 (1988).

[9] N. A. Spenley, M.E. Cates, and T.C. B. McLeish, Phys. Rev. Lett. 71, 939 (1993).

[10] M. Britton and P. Callaghan, Phys. Rev. Lett. 78, 4930 (1997).

[11] M. R. López-González et al., Phys. Rev. Lett. 93, 268302 (2004).

[12] G. Marrucci, J. Non-Newtonian Fluid Mech. 62, 279 (1996).

[13] G. Ianniruberto and G. Marrucci, J. Rheol. (N.Y.) 45, 1305 (2001); J. Non-Newtonian Fluid Mech. 102, 383 (2002).

[14] D. W. Mead, R. G. Larson, and M. Doi, Macromolecules 31, 7895 (1998).

[15] J. Bent et al., Science 301, 1691 (2003).

[16] S. T. Milner, T.C.B. McLeish, and A.E. Likhtman, J. Rheol. (N.Y.) 45, 539 (2001).

[17] R.S. Graham, A.E. Likhtman, and T.C.B. McLeish, J. Rheol. (N.Y.) 47, 1171 (2003).

[18] V.R. Mhetar and L.A. Archer, J. Polym. Sci. B 38, 222 (2000); J. Non-Newtonian Fluid Mech. 81, 71 (1999).

[19] F. Brochard-Wyart and P. G. deGennes, Langmuir 8, 3033 (1992).

[20] A. Ajdari et al., Physica (Amsterdam) 104A, 17 (1994).

[21] V. R. Mhetar and L.A. Archer, Macromolecules 31, 6639 (1998); 31, 8607 (1998).

[22] Y.M. Joshi, A. K. Lele, and R. A. Mashelkar, Macromolecules 34, 3412 (2001).

[23] P. Tapadia and S. Q. Wang, Phys. Rev. Lett. 96, 016001 (2006).

[24] P. Tapadia, S. Ravindranath, and S. Q. Wang, Phys. Rev. Lett. 96, 196001 (2006).

[25] P.E. Boukany and S. Q. Wang, J. Rheol. (N.Y.) 51, 217 (2007).

[26] Y. T. Hu and L. Wilen, J. Rheol. (N.Y.) 51, 275 (2007).

[27] I. Cohen et al., Phys. Rev. Lett. 97, 215502 (2006).

[28] R. G. Larson, Rheol. Acta 31, 213 (1992).

[29] S. Kumar and R. G. Larson, J. Non-Newtonian Fluid Mech. 95, 295 (2000).

[30] Y.W. Inn, K.F. Wissbrun, and M. M. Denn, Macromolecules 38, 9385 (2005)

[31] C. Sui and G. B. McKenna, Rheol. Acta 46, 877 (2007).

[32] V. R. Mhetar and L. A. Archer, J. Rheol. (N.Y.) 40, 549 (1996).

[33] R. B. Bird, R. C. Armstrong, and O. Hassager, Dynamics of Polymeric Liquids (Wiley, New York, 1987), Vol. 1. 